\title{
Microcontroller-based Wireless Heart Rate Telemonitor for Home Care
}

\author{
Sanjay Verma, Namit Gupta, \\ Electronics Department, S.V.I.T.S. INDORE/R.G.P.V. University Bhopal, India
}

\begin{abstract}
Homecare is the provision of health care services to patients in their own home. One of the main purposes of homecare telemedicine is to develop a wireless, low-cost and use-friendly system which allows patients to measure their own vital signs, such as heart rate and temperature, and provide the health care professionals with the facility to remotely monitor the patient's vital signs quickly and easily. The gadget would then activate a GSM modem (SIM based) and also a GPS (global positioning system). The gadget would then take out the location reading from the GPS. Other vital information such as Heart Rate and temperature is taken and sent to predefined numbers. The receiver will get an SMS which will contain information of the senior person with his/her position co-ordinates and his/her current status (Heart Rate). An onscreen display will start scrolling the person's name, address and contact details so that people who try to help such a person get the complete information from the device.
\end{abstract}

Keywords - PIC Microcontroller, LM35, GPS, GSM, Heart Rate sensor.

\section{INTRODUCTION}

Today, the medical field is faced with growing public concerns and government demands for reform. The outrage is directed at the high costs of quality health care and the inability of healthcare specialists to provide adequate medical services to rural populations. The availability of prompt and expert medical care can meaningfully improve health-care services at understaffed rural and remote areas [1-3]. In distant regions of the country the degree of development of healthcare providing services has not reached the appropriate level to adequately address the health care needs of the populations in these areas. A mobile monitoring system utilizing Short Message Service with low-cost hardware equipment has been developed and implemented to enable transmission of the temperature and Heart Rate signal of a patient [10]. At the consultation unit, dedicated application software is required to manage the follow of SMS messages from the Mobile and display the temperature and Heart Rate of the patient. The special of this device is it is not only measure Heart Rate and temperature measurement, but also will display the measured Heart Rate in beat per minute. The device consists of electronic circuit system, embedded system which is microcontroller program. The Heart Rate monitoring systems consists infrared LED, photo transistor as a sensing device for the Heart Rate system. Conventional telemedicine systems using Public Switched Telephone Network (PSTN) land lines are already available to enable a doctor to monitor a patient remotely for home care or emergency applications [5], [6]. Also, the mobile phone has been recognized as a possible tool for telemedicine since it became commercially available. This system which allows patients to measure their own vital signs, such as Heart Rate, temperature and provide the health care professionals with the facility to remotely monitor the patient's vital signs quickly and easily, vital signs can be transmitted from an ambulance to a hospital in a store-and forward mode [7], [8], or in real-time mode [9].

A PIC16C774A microcontroller was then interfaced to the wearable Heart Rate signal to collect the data from these devices, process them, store them and feed them to a transmitter. A high performance RF Module Tri-Band GSM/GPRS 900/ 1800/ $1900 \mathrm{MHz}$ transmitter and receiver were then used to Wirelessly transmit and receive the vital sign data from the micro-controller to the consultation unit, dedicated application is required to manage the follow of SMS messages from the Mobile and display the temperature, location and Heart Rate of the patient". 


\section{SYSTEM CONCEPT}

The proposed mobile telemedicine (monitoring) system is shown in Fig 1

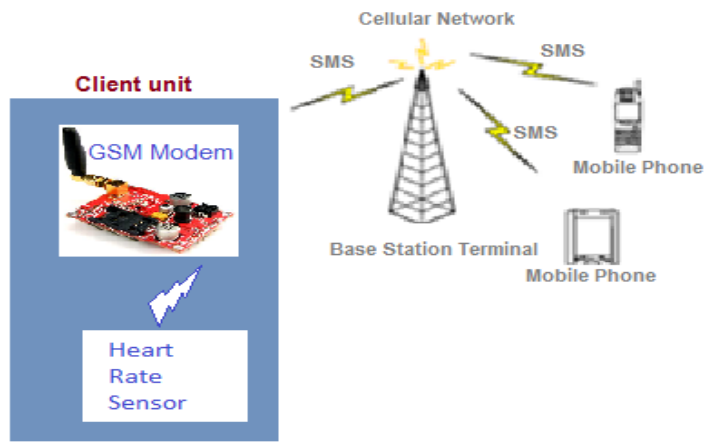

Fig 1.Mobile Monitoring and Measurement System

The patient (client) and the health care professional (server) can be located anywhere in the globe where there is GSM (cellular) network coverage. The patient's Heart Rate and temperature, and other vital signs if desired, can be acquired by the patient himself under follow up scenario for example, or the patient can be assisted by a family member or a health care professional in more serious cases depending on the particular patient's case [4].The signal acquisition process is performed by attaching the Heart Rate electrodes and the temperature sensor to the patient's body at designated places as is normally done in a typical similar set-up. The client unit communicates with the mobile phone via an GSM modem connection, which can be established through a UART. The mobile phone in- turn submits a series of SMS messages that contains the acquired data to the cellular network by communicating with the serving cellular base station. When the SMS messages reach their destination mobile phone (or PDA), they are either downloaded via and RS232 connection by a special software running on a laptop, or they can be imported by a mobile application running on the phone (or PDA) [4].

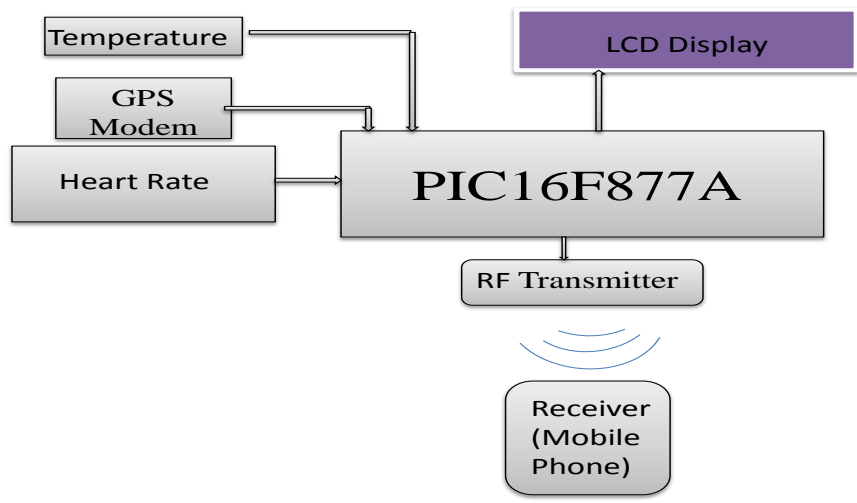

Fig 2.Block Diagram of Heart Rate and Temperature Measurement System (Client Unit and Server Unit)

\section{THE USER UNIT}

The client unit is comprised of vital-signs signals acquisition module and mobile phone. The core of the signal acquisition module is a microcontroller (PIC16F877) as shown in Fig 2.

The microcontroller acquires the amplified and conditioned signals, and then performs the interface with the mobile phone using the supported standard AT commands. 


\subsection{Heart Rate Sensor}

Heart beat sensor is designed to give digital output of heat beat when a finger is placed on it. When the heart beat detector is working, the beat LED flashes in unison with each heart beat. This digital output can be connected to microcontroller directly to measure the Beats per Minute (BPM) rate. It works on the principle of light modulation by blood flow through Fig 3. At each pulse [13].

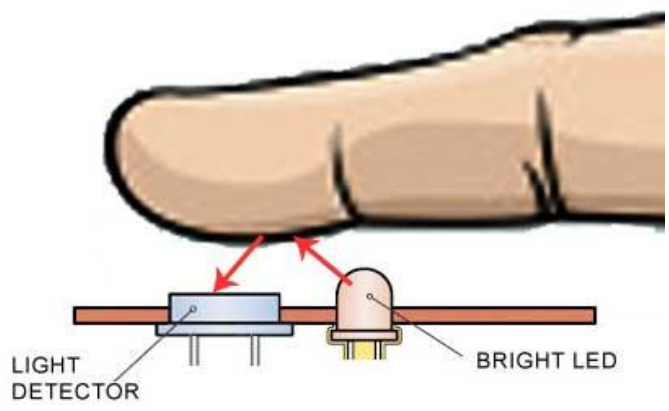

Fig 3.Working Operations

\subsection{GPS Receiver Sensor}

This is a high gain GPS Receiver (5V Serial) with 4pin. Receiver is made with third generation POT (Patch Antenna on Top) GPS module. The built in 3V3 to 5V level convertor enable us to interface with normal 5V Microcontrollers. The 4 Pins are 5V, TX, RX, and GND. Yes, there is no setting required, just plug in to the power (5v), your data (NMEA 0183) is ready at TX pin! This is a standalone 5V GPS Module and requires no external components .It is built with internal RTC Back up battery. It can be directly connected to Microcontroller's UART. With the use high gain GPS engine providing a solution that high position and speed accuracy performances as well as high sensitivity and tracking capabilities in urban conditions \& provides standard NMEA0183 strings in "raw" mode for any microcontroller. The module provides current time, date, latitude, longitude, speed, altitude and travel [12].

\subsection{GSM Modem}

GSM/GPRS Modem-TTL (5V is built with Tri-band GSM/GPRS engine, works on frequencies EGSM $900 \mathrm{MHz}$, DCS $1800 \mathrm{MHz}$ and PCS $1900 \mathrm{MHz}$ It is very compact in size and easy to use as plug in module. The Modem is coming with 5V TTL interface, which allows you to connect directly to 5V microcontroller. The baud rate is configurable from 9600-115200 through AT command. The GSM/GPRS TTL Modem is having internal TCP/IP stack to enable you to connect with internet via GPRS. It is suitable for SMS as well as DATA transfer application in M2M interface.

You need only two wires (Tx, Rx) except Power supply to interface with microcontroller. Using this modem, you can send SMS, data and read SMS through simple AT command .Inbuilt Powerful TCP/IP protocol stack for internet data transfer over GPRS. Input Voltage: 9-12VDC [12].

\subsection{Body Temperature Sensor}

We used a special rapid, low-cost, integrated-circuit temperature sensors. The LM35 sensor thus has an advantage over linear temperature sensors calibrated in ${ }^{\circ}$ Kelvin, as the user is not required to subtract a large constant voltage from its output to obtain convenient Centigrade scaling. The LM35 sensor does not require any external calibration or trimming to provide typical accuracies of $\pm 1 / 4^{\circ} \mathrm{C}$ at room temperature and $\pm 3 / 4^{\circ} \mathrm{C}$ over a full -55 to $+150^{\circ} \mathrm{C}$ temperature range. It can be Operates from 4 to 30 volts. As it draws only $60 \mu \mathrm{A}$ from its supply, it has very low self-heating, less than $0.1^{\circ} \mathrm{C}$ in still air. The LM35 is rated to operate over a $-55^{\circ}$ to $+150^{\circ} \mathrm{C}$ temperature range. We interface the temperature sensor to the PIC16F877A microcontroller using the ADC port on the microcontroller [4].

\subsection{PIC16F877A Microcontroller}

The PIC 16F877 is an 8-bit microcontroller, which has an on-chip eight channel 10-bit Analog-toDigital Converter (ADC).First we detect fall down using accelerometer and fed to the $\mathrm{I}^{2} \mathrm{C}$ ports. The amplified and conditioned Heart Rate signal is fed to input port RB0 (INT) of the microcontroller. Also, upon command, the microcontroller reads the temperature sample stored in the RAM of the LM35 through the ADC port RA0. It is then converted and stored in the PIC16F877 memory as two 8-bit unsigned integers (0-255).Fig 4.shows a flowchart of the microcontroller program. 
After completion of signals acquisition, the microcontroller constructs the SMS messages and packs the data samples in these messages to the desired length, then communicates with the mobile phone using at-commands on its GSM modem port to send the message(s).

A complete system can therefore be built using one MCU chip and a few I/O devices such as a keypad, display and other interfacing circuits. Most of the pins are for input and output, and arranged as 5 ports: PORTA (5pins), PORTB (8pins), PORTC (8pins), PORTD (8pins) and PORTE (3 pins), total of 32 I/O pins [5], [14].

\subsection{LCD Display}

The Model JHD 162A Series LCD is the typical standard HD44780 type of LCD with 16characters $\mathrm{x} 2$ row LCD module. Since this project the Heart Rate, temperature, adders and contact no to display; therefore, a LCD module is necessary.

\subsection{AT commands}

The AT commands are standard control tools based on GSM to establish communication with the mobile GSM phone or modem [112. The commands set consist of strings, which will enable the exchange of serial data, according to certain syntax rules, between the mobile and the microcontroller at the client unit, and the laptop or PC at the server. As an example: AT \r\n check whether communication is established."ATE0 \r|n" is echo off. AT+CMGF=1 $\mathrm{r} \backslash \mathrm{n}$ switch to text mode. AT+CMGS="Mobile Number"' $\mathbf{r} \backslash \mathrm{n}>$ is for send the SMS to the mobile number."AT+CMGS=140", is a command for sending a SMS message, where "AT" is a prefix used for all commands, "CMGS" is a description to the kind of task to be performed, and "140" is the message length. Similarly, when a message is received by the laptop according to the AT command "AT $+\mathrm{CMGR}=$ "mobile number" $|r| n$, for read the SMS with message index number stored in the SIM card. The program will be able to divide the message contents in order to extract the binary 8-bit samples to display the latitude longitude, temperature and Heart Rate data in the User Data (UD) part of the SMS(s) [12].

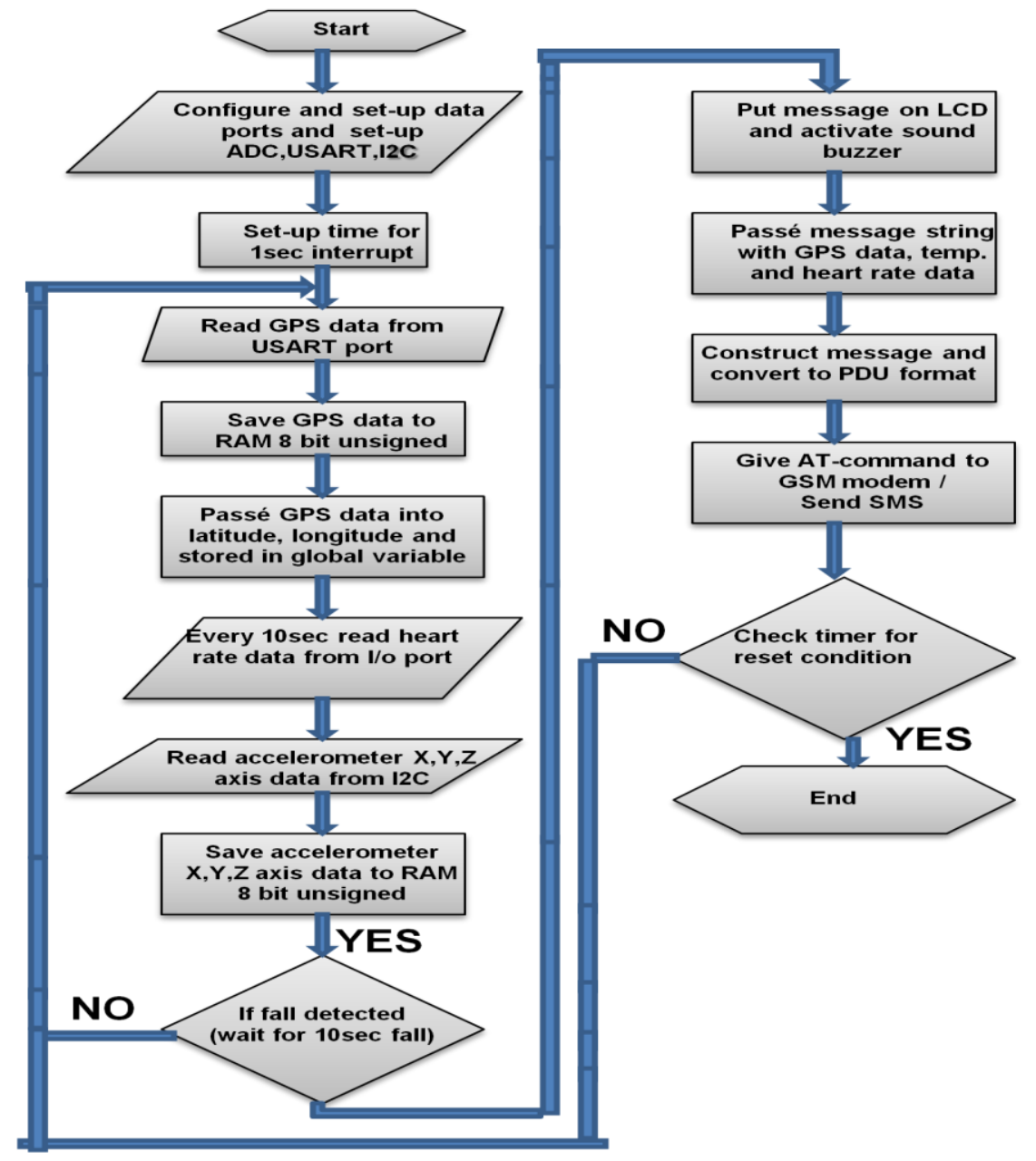

Fig 4.Microcontroller Software Flowchart 


\section{THE CONSULTATION/SERVER UNIT}

The platform or Operating System (OS) used to run the application software at receiving device will influence the choice of the preferred programming language used in implementing the software. The smartphone we have used was the Motorola mobile phone. Capturing the data through SMS message(s) decoding them, and extracting the user data part .

The software decodes each SMS and extracts the time and date, originating mobile number, and the transmitted patient's temperature and Heart Rate samples in the payload. Figure 5 shows a screen interface of the application software running and displaying a list of received and decoded SMS messages [4].

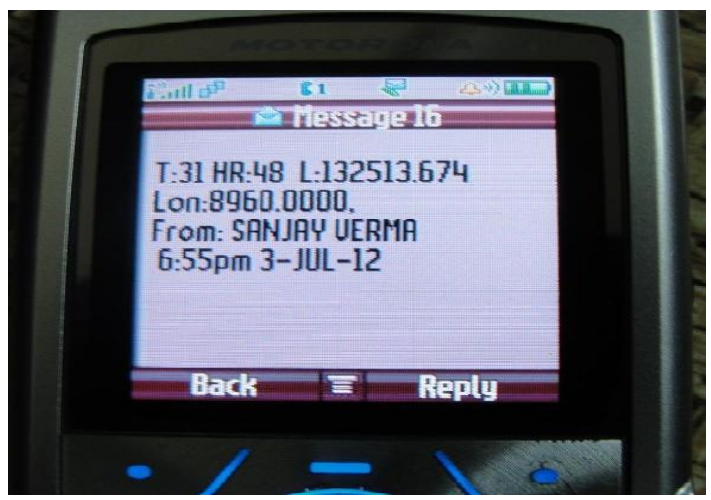

Fig 5.Motorola smart-phone displaying a Heart Rate, body temperature and latitude, longitude

The software converts the data in the message from binary to ASCII and displays the contents of the message as shown Figure 5. The first three digits represent body temperature (were obtained from two bytes) with an implicit decimal point after the first two digits from the left. Each Heart Rate sample can take a value between 0 and 255 as a maximum since we originally used eight bit accuracy in our ADC Also, the application software has a menu button, which allows for plotting the Heart Rate sample points and displaying the Body temperature reading contained in the selected SMS message from the list. Figure 5 shows the Motorola smart phone displaying a Heart Rate with a body temperature reading at the top.

\section{EXPERIMENTAL RESULTS}

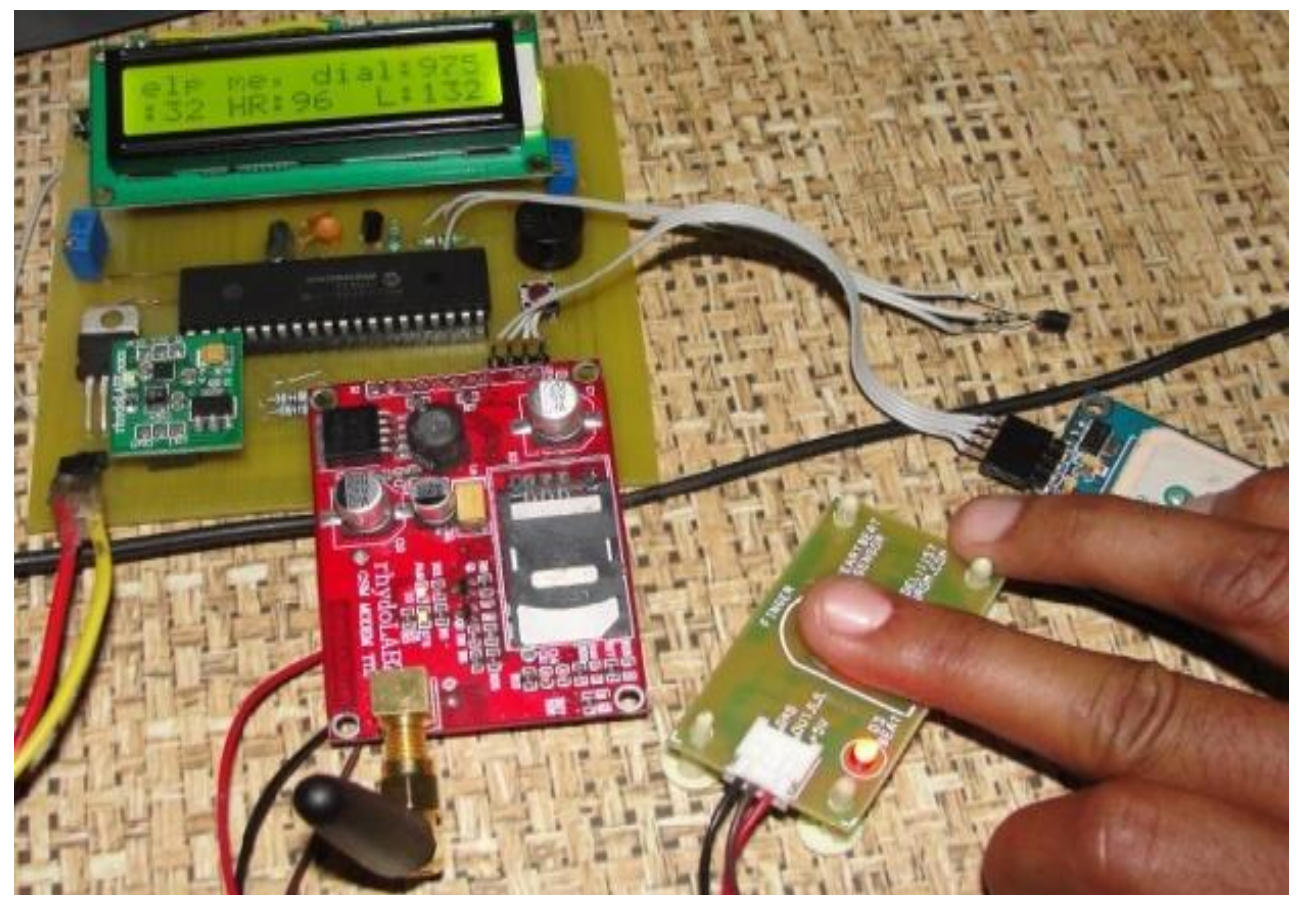

Fig 6.Fall down then Display Heart Rate, Temperature and latitude, longitude 


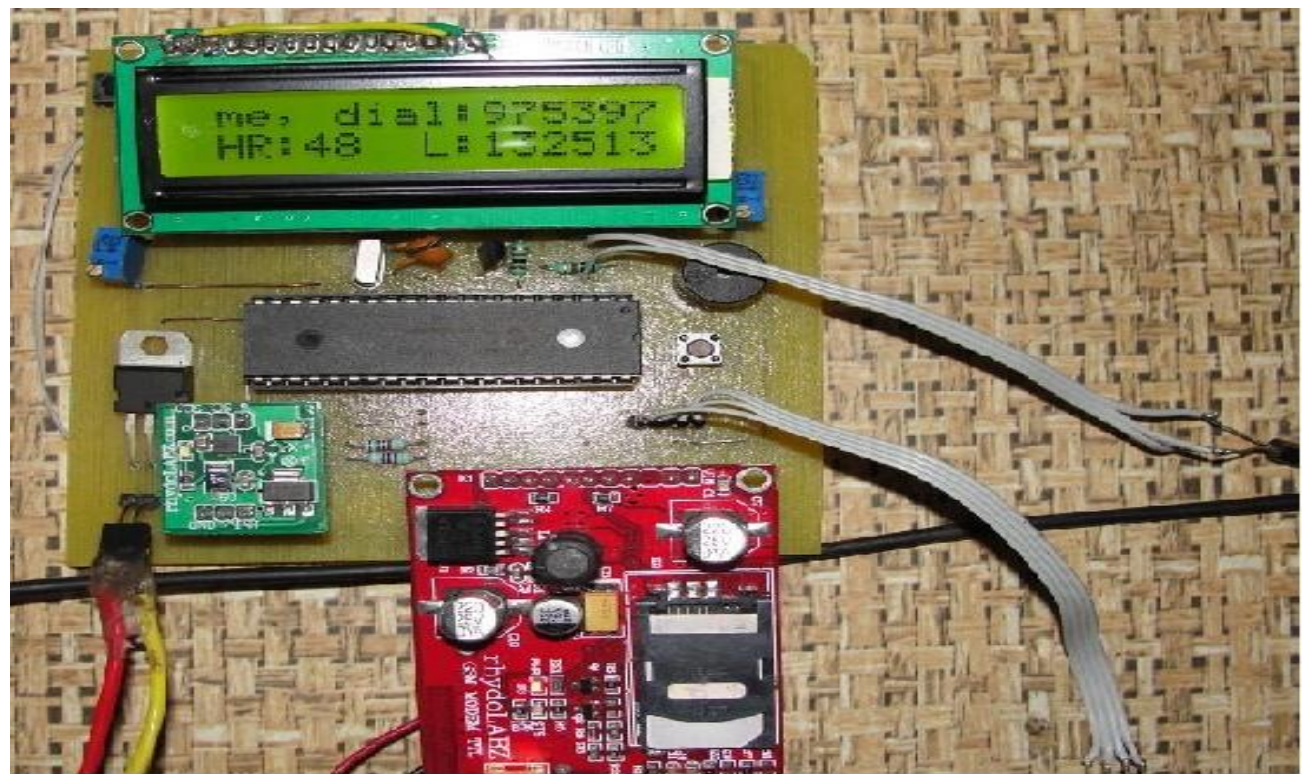

Fig 7.Fall down then Display Heart Rate, Temperature and latitude, longitude

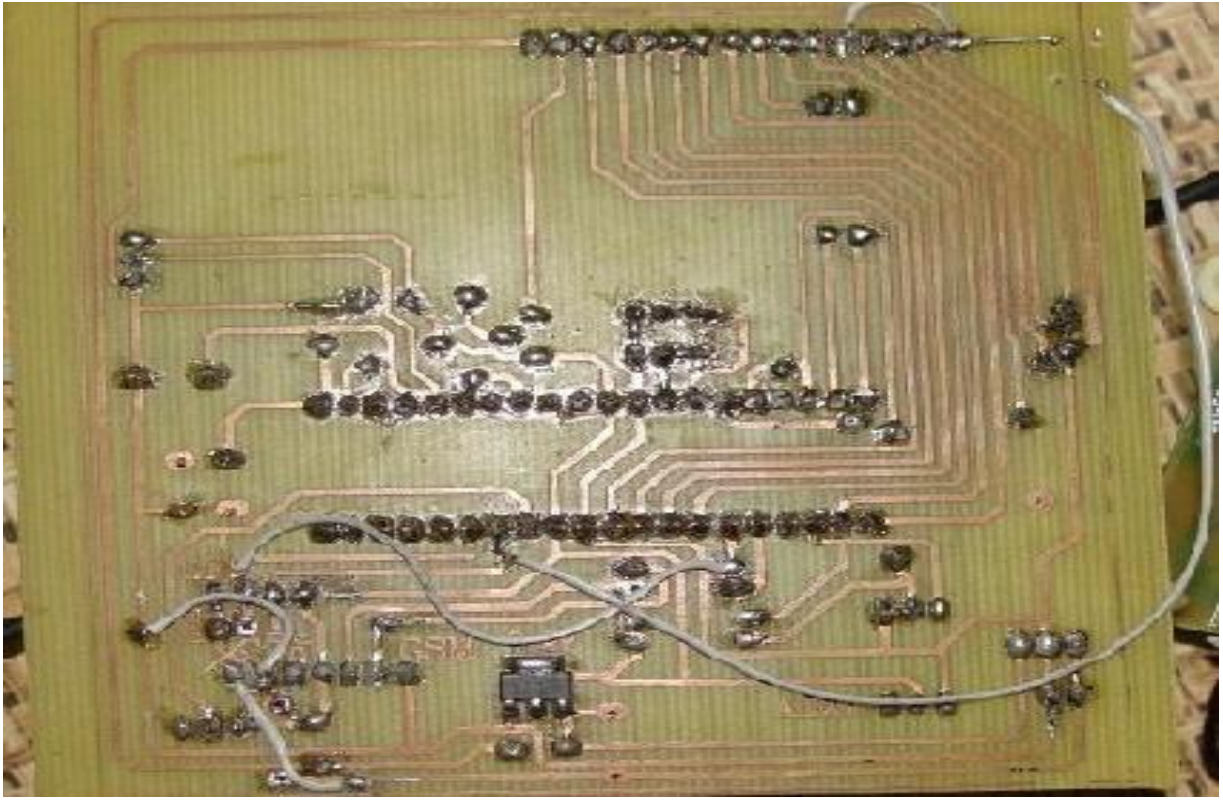

Fig 8.Backside of Implemented hardware system

\section{CONCLUSION}

A low cost mobile patient monitoring system that utilizes Short Messaging Service (SMS) was designed, developed, and tested. An Infrared temperature sensor was integrated with a three lead Heart Rate monitor (client unit) on a cellular (mobile) phone platform, which can be considered as a real time transmission mode. Application software is required at the receiving mobile device (consultation unit) to decode the signal SMS messages and plot the Heart Rate and display the body temperature. The new system has a significantly reduced size and weight, which improves its versatility and mobility. Besides, SMS can be the most suitable, if not the only, method of data transmission in emergency situations in remote area where broadband data communications (like GPRS, EDGE ... etc.) are not available. Fig2 shows the steps performed by the microcontroller to retrieve the digital representation of Heart Rate and Temperature transmit them to the receiver using the wireless transmission system outlined above. In future work more powerful transmitters with higher range will be used and the flexibility to use the internet to send the data to the receiving site will be fully explored. 


\section{Journal Papers:}

\section{REFERENCES}

[1] Long J (1995) Quality in the US. health cure system. Am J Med Qual 1O(4): 221-224.

[2] Board of Directors of the American Medical Informatics Association (1997). A proposal to improve quality. Increase efficiency, and expand access in the U.S. health care system (1997). J Am Med Inform Assoc 4(5):340-341, Sept. 1997.

[3] Bashshur R L (1995). relemedicine erects: cost. quality. And access. J Med Syst 19(2):81-91, 1995.

[4] Ashraf A Tahat developed by 'Body temperature and electrocardiogram monitoring using an SMS-based telemedicine system', School of Electrical Engineering Princess Sumaya University for Technology Amman, Jordan

[5] U. Patel, C. Babbs, A computer based automated telephonic system to monitor patient progress in home setting, Jornal of Medical Systems, vol. 16(2), 1992, 101-112.

[6] G. Coyle, L. Boydell, L. Brown, Home telecare for the elderly,Journal of Telemedicine and. Telecare, vol. 1, $1995,183-185$.

[7] P. Giovas, D. Papadoyannis, D. Thomakos, et al., Transmission of electrocardiograms from a moving ambulance, Journal of Telemedicine and Telecare, vol. 4, 1998, 5-7.

[8] T. Samaras, S. Karavasiliadou, E. Kouidi, et al.Transtelephonic electrocardiographic transmission in the preparticipation screening of athletes, International Journal of Telemedicine and Applications, vol. 1, 2008, 1-4.

[9] K. Shimizu, Telemedicine by mobile communication, IEEE Engineering in Medicine. Biology Magazine. Vol. 18(4), $1999,32-44$.

\section{Books:}

[10] G. Bodic, "Mobile messaging technologies and services", (West Sussex, England: John Wiley\& Sons, 2005).

[11] S. Guthery, M. Cronin, Mobile Application Development with SMS and the SIM Toolkit (New York, NY: McGraw-Hill , 2002)

\section{Web site:}

[12] http://www.rhydolabz.com

[13] http://www.sunrom.com

[14] http://www.microchip.com/ 